\title{
Experimental study on MRI evaluation of the course of cervical spinal cord injury
}

\author{
K Ohta, ${ }^{* 1}$ Y Fujimura ${ }^{1}$, M Nakamura ${ }^{1}$, M Watanabe ${ }^{1}$ and Y Yato ${ }^{1}$ \\ ${ }^{1}$ Department of Orthopaedic Surgery, School of Medicine, Keio University, Tokyo, Japan
}

\begin{abstract}
Study Design: An experimental study was conducted to evaluate MRI signal changes within the spinal cord after the injury in rats.

Objectives: To clarify the significance of MRI signal changes in the injured cervical cord. Setting: Tokyo, Japan.

Methods: Cervical spinal cord injury was produced in rats by placing a $20 \mathrm{-g}$, or $35 \mathrm{-g}$ weight on exposed dura at the C6 level for $5 \mathrm{~min}$ ( $20 \mathrm{~g}$ - or $35 \mathrm{~g}$-compression group). Motor function was evaluated by the inclined-plane method at 2, 7 and 28 days after the injury. T1- and T2weighted images were produced by the spin-echo method with a static magnetic field strength of 2.0 tesla, at 2 and 28 days after the injury, and then the histopathological examinations were performed.

Results: In the 20-g compression group, which recovered from the paralysis at 28 days, MR images were $\mathrm{T} 1$ iso signal/T2 high signal 2 days after the injury and T1 iso signal/T2 high signal after 28 days. The changes in MRI signal 2 and 28 days represented edema and gliosis, respectively. In the $35-\mathrm{g}$ compression group, which incompletely recovered from paralysis at 28 days. MR images were T1 iso-signal/T2 low signal surrounded by high signal 2 days after the injury and T1 low/T2 high signal at 28 days. The MRI signal changes 2 and 28 days reflected hemorrhage with edema and cavities, respectively.

Conclusion: T2 low signal of the spinal cord observed early after injury reflects hemorrhage and may serve as an indicator of a poor prognosis. T1 low/T2 high signals from the subacute to chronic period indicated persistence of paralysis and limited recovery of function.
\end{abstract}

Keywords: cervical spinal cord; incomplete injury; magnetic resonance imaging; histopathology

\section{Introduction}

MRI has been widely used in the diagnosis of spinal cord injury in recent years, and its usefulness is indisputable. Studies have been conducted in regard to MRI findings, especially comparing signal changes and histological findings in the injured cord. Duncan et $a l^{1}$ created an injury model with different degrees of severity and compared the in vitro MRI findings and histopathological findings $1 \mathrm{~h}$ after the injury. Hackney et $a l^{2}$ created an injury model with different degrees of severity, described the in vitro MRI findings $4 \mathrm{~h}$ after the injury, and claimed that it was possible to clearly distinguish between white matter and gray matter. Becerra et $a l^{3}$ used the spinal cord of patients who had died of spinal cord injuries and compared the in vitro MRI findings and pathology findings.

However, few investigators have assessed the course of pathological changes over time. In our study, a

*Correspondence: K Ohta, Department of Orthopaedic Surgery, School of Medicine, Keio University, 35 Shinanomachi, Shinjuku-ku, Tokyo, 160 Japan model of cervical cord injury with different degrees of severity was created, and in vivo MRI signal changes in the cord were compared with changes in impairment of motor function and histopathological changes, in order to elucidate the course of pathological changes in the cervical cord over time.

\section{Materials and methods}

Incomplete spinal cord injury model

A spinal cord model injury was created in 50 female Wistar rats weighing $250-300 \mathrm{~g}$ by the weightplacement method, according to the procedure described by Holtz et al. ${ }^{4}$ After anesthetizing the animals with pentobarbital $(50 \mathrm{mg} / \mathrm{kg}$ IP), cervical cord injury was created by performing laminectomy of cervical vertebrae 5 and 6 (C5 and C6) under a microscope and placing a 20 or $35 \mathrm{~g}$ weight on the dura at the C6 level for $5 \mathrm{~min}$ to produce a $20-\mathrm{g}$ compression group and a $35-\mathrm{g}$ compression group. ${ }^{5,6}$ The bottoms of the weights used in this study were 
made concave and had a bottom surface area of $6.6 \mathrm{~mm}^{2}(3.0 \times 2.2 \mathrm{~mm})$ so that uniform compression would be applied to the spinal cord. The animal's neck and the spinous process of the second thoracic vertebra were fixed as a means of further restricting movement of the cervical cord during compression. A sham group in which laminectomy alone was performed with no application of compression was also prepared as a control.

\section{Evaluation of motor function}

Motor function was evaluated 2, 7 and 28 days after the injury by the inclined-plane method of Rivlin et $a l^{7}$ as the mean of the three maximum angles at which the animal was able to maintain its trunk (cling) on an inclined platform for $5 \mathrm{~s}$.

\section{MRI evaluation}

The rats (20-g compression group: 2 days after the injury $n=10,28$ days after $n=10 ; 35$-g compression group: 2 days after injury $n=10,28$ days after $n=10$; sham group: $n=10$ ) were anesthetized in the manner described above and fixed in the prone position on a fixation stand prepared for MRI. A $5 \times 8-\mathrm{cm}$ surface coil positioned so that it closely fit the neck was used as the receiver. Midsagittal images and cross-sectional images were produced at the level of the body of C6 with a 2.0-T Bruker superconducting unit by the spinecho method at a slice thickness of $2.8 \mathrm{~mm}$ under T1weighted conditions were TR500, TE17 and 4 NEX, and T2-weighted conditions were TR3000, TE100 and 4 NEX.

T1/T2 signal intensity ratios were calculated to evaluate internal signal changes in the cervical cord by MRI more quantitatively. The signal intensity ratios were obtained by using copper sulfate as the reference material and dividing the internal signal intensity of the cervical cord by the internal signal intensity of the copper sulfate.

\section{Histopathological examination}

After the MRI evaluations, transcardiac perfusion was performed with $4 \%$ paraformaldehyde, and the cervical cord was removed en bloc. Following post-fixation with the same solution, frozen sections were prepared at the C6 cord level and stained with hematoxylin-eosin.

In addition, in order to assess spinal cord edema as a result of damage to the blood-spinal cord barrier, $1 \%$ Evans blue $(10 \mathrm{ml} / \mathrm{kg}$ body weight) was injected into the femoral vein, and transcardiac perfusion fixation with $4 \%$ paraformaldehyde was performed 30 min later. After excising the cervical cord en bloc and post-fixing it with the same solution, frozen sections were prepared at the C6 level, and the sections were inspected for Evans blue leakage by $G$ excitation $(465-550 \mathrm{~nm})$ with a fluorescence microscope.

\section{Immunohistochemical studies}

After the MRI evaluations, immunohistochemical studies were performed using an antibody to glial fibrillary acidic protein (GFAP) to identify the change in astrocytes. Staining was performed with a Histyofine Sab-Po (Multi) kit. The specimens were exposed to anti-human glial fibrillary acidic protein (DAKO, clone $6 \mathrm{~F} 2$ ) for $2 \mathrm{~h}$ at room temperature and colorized with $\mathrm{DAB}$ for $5 \mathrm{~min}$ at room temperature.

\section{Statistical analysis}

The results are expressed as means \pm SD. Differences in motor functional ability and changes in signal ratios among the groups were compared using one-way ANOVA followed by Fisher's protected least significant differences (PLSD) test. Statistical significance was defined as $P<0.05$.

\section{Results}

\section{Evaluation of motor function}

Motor dysfunction in terms of loss of clinging ability was not observed in the sham group until $82 \pm 4^{\circ}$ had been reached. In the $20 \mathrm{-g}$ compression group, severe paralysis of the forelimbs was observed 2 days after the injury, and clinging ability on the inclined platform had decreased to $55 \pm 6^{\circ}$. Motor function later recovered, and by 28 days the animals were able to run, and their clinging ability had recovered to $80 \pm 2^{\circ}$. In the 35-g compression group, paralysis was observed in both the forelimbs and the hind limbs 2 days after the injury. The animals were unable to walk, and their clinging ability on the inclined platform had decreased to $30 \pm 3^{\circ}$. It improved to $55 \pm 4^{\circ} 7$ days later, and to $65 \pm 7^{\circ}$ by 28 days, but the forelimb paralysis persisted.

\section{MRI findings}

No signal changes in the spinal cord were observed in the sham group on either the T1-weighted or the T2weighted images. In the $20-\mathrm{g}$ compression group, the T1-weighted images were iso-signal and did not show any changes either 2 or 28 days after the injury. On the T2-weighted images, diffuse high signals centered on the compressed area were observed 2 days after the injury, and high signals localized to the dorsal spinal cord in the compressed area were seen at 28 days (Figure 1). In the 35-g compression group, the T1weighted images were iso-signal 2 days after the injury, but they showed a clearly demarcated low-signal area in the center of the spinal cord in the compressed area after 28 days. On the T2-weighted images, there was a low-signal area at the center of the spinal cord in the compressed area with a surrounding diffuse high signal 2 days after the injury, and after 28 days a clearly demarcated high signal localized from the center to the dorsal area of the spinal cord was seen in the compressed portion (Figure 2). 

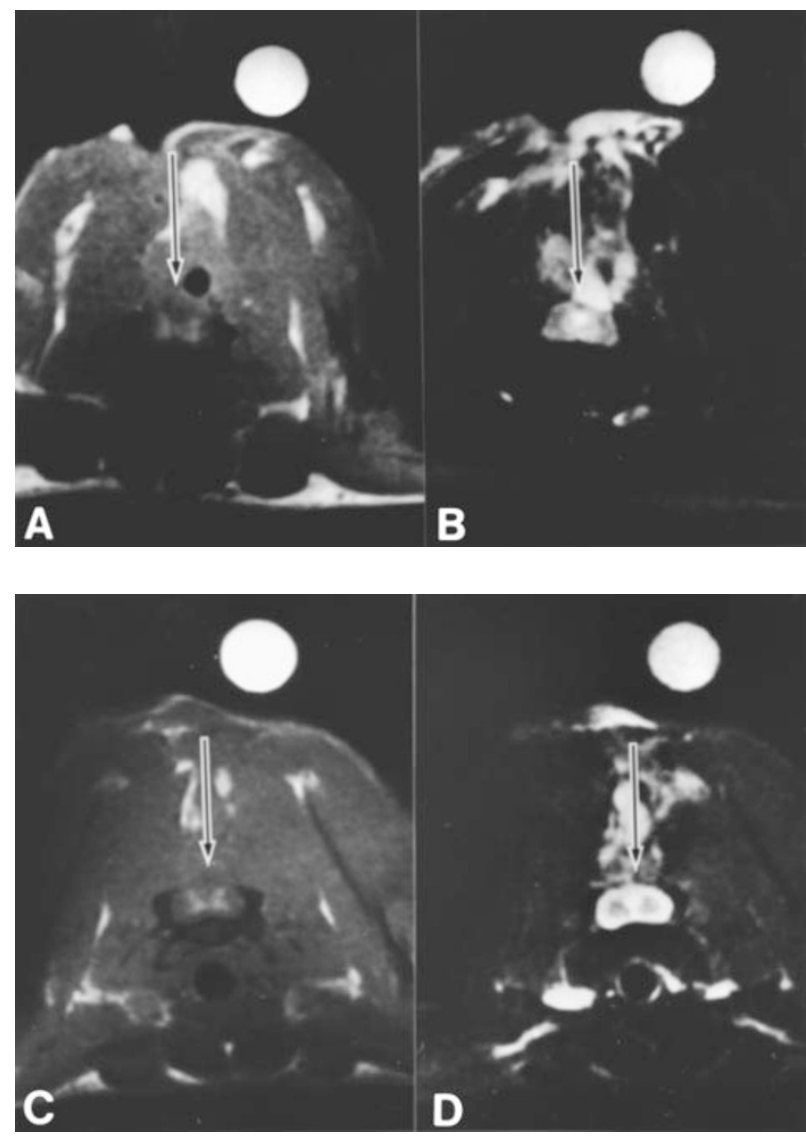

Figure 1 MRIs in the 20-g compression group. Iso signal on T1-weighted image (A) and diffuse high signal on T2weighted image (B) were observed in the compressed spinal cord 2 days after the injury (arrow). Iso signal on T1weighted image $(\mathbf{C})$ and localized high signal on T2-weighted image (D) were observed in the compressed spinal cord 28 days after the injury (arrow)

The changes in $\mathrm{T} 1 / \mathrm{T} 2$ signal intensity ratios over time in the 20 -g and the $35-\mathrm{g}$ compression groups can be summarized as follows: in the 20 -g compression group, the findings were $\mathrm{T} 1$ iso-signal/T2 high signal 2 days after the injury and $\mathrm{T} 1$ iso-signal/T2 high signal after 28 days; in the 35-g compression group, the findings were T1 iso-signal/T2 high signal 2 days after the injury, and T1 low signal/T2 high signal after 28 days (Table 1).

\section{Histopathological findings}

In the 20 -g compression group, changes that seemed to represent edema of the spinal cord were observed 2 days after the injury, but changes that seemed to represent reactive gliosis were observed in the dorsal column after 28 days. In the 35 -g compression group, hemorrages were observed 2 days after the injury, predominantly in the dorsal gray matter, and spongiform change was noted in the white matter. After 28
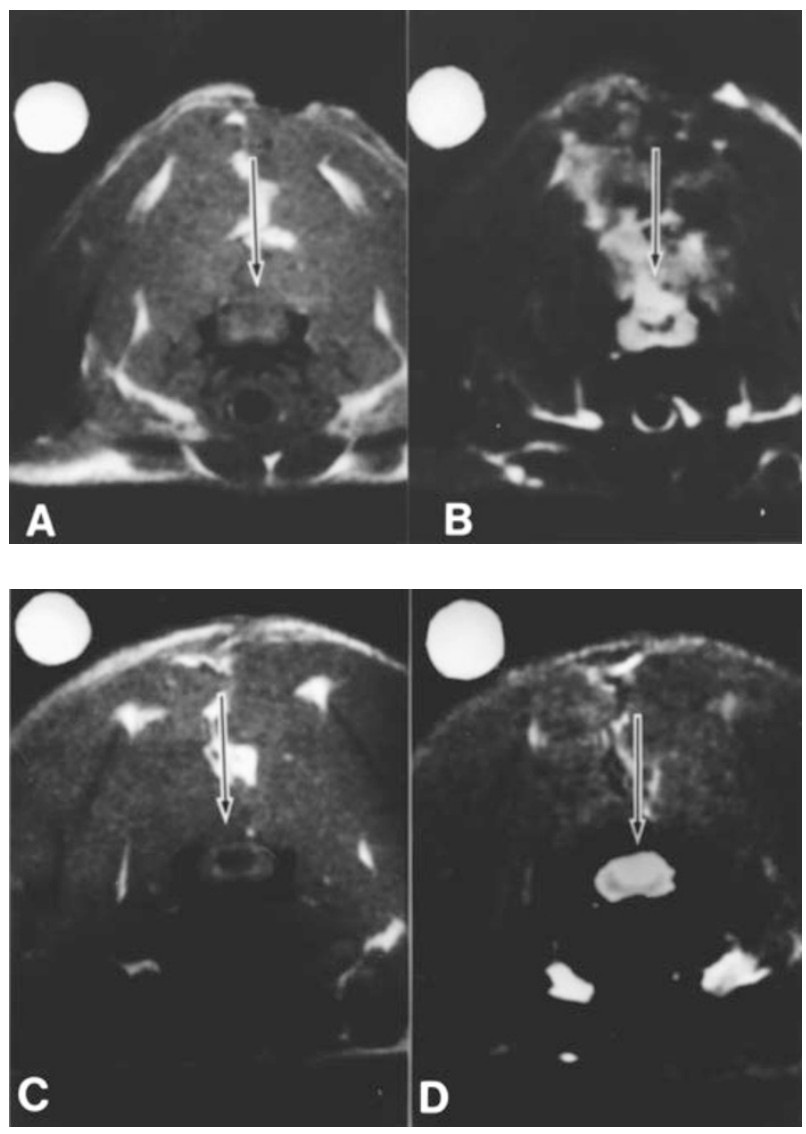

Figure 2 MRIs in the 35-g compression group. Iso signal on T1-weighted image (A) and low signal with a surrounding diffuse high signal on T2-weighted image (B) were observed in the compressed spinal cord 2 days after the injury (arrow). Clearly demarcated low signal on T1-weighted image (C) and high signal on T2-weighted image (D) were observed in the compressed spinal cord 28 days after the injury (arrow)

Table 1 Changes in signal intensity ratios

\begin{tabular}{lcc}
\hline & $\begin{array}{c}T 1 W \\
(\text { mean } \pm \text { SD) }\end{array}$ & $\begin{array}{c}T 2 W \\
(\text { mean } \pm \text { SD) }\end{array}$ \\
\hline Sham group $(n=10)$ & $0.47 \pm 0.02$ & $1.09 \pm 0.05$ \\
20 g compression group & & \\
$\quad$ 2 days $(n=10)$ & $0.48 \pm 0.06$ & $1.69 \pm 0.20^{*}$ \\
$\quad$ 4 weeks $(n=10)$ & $0.51 \pm 0.16$ & $1.82 \pm 0.28^{*}$ \\
35 g compression group & & \\
$\quad$ 2 days $(n=10)$ & $0.47 \pm 0.09$ & $1.51 \pm 0.15^{*}$ \\
4 weeks $(n=10)$ & $0.18 \pm 0.06^{*}$ & $2.13 \pm 0.17^{*}$ \\
\hline
\end{tabular}

* Significantly different $(P<0.05)$ from the value of the Sham group as determined by Fisher's PSLD test

days, cavity formation and surrounding reactive gliosis were observed (Figure 3).

Examination for damage to the blood-spinal cord barrier with Evans blue showed that the dye had accumulated within the blood vessels in the sham 
group. In the 20-g compression group, however, it had leaked out into the spinal cord parenchyma 2 days after the injury, but after 28 days hardly any leakage was detected (Figure 4).

\section{Immunohistochemical studies}

Immunohistochemical studies for the identification of astrocytes using GFAP antibody indicated no detectable changes in the sham group, 2 days after the injury. Twenty-eight days after the injury in the 20 -g compression group showed astrocyte proliferation primarily in the dorsal column (Figure 5), and 35-g compression group showed astrocyte proliferation around the cavity formation.

\section{Discussion}

The outcome of cervical cord injury varies considerably with its severity, and while MRI studies of the pathology have been performed, there have been no studies comparing the MRI findings with the histopathological findings. ${ }^{8}$ Based on the clinical picture and the MRI findings, Kulkarni et al divided the MRI signal changes in the spinal cord in the acute stage into three patterns and reported that low signals on the T2-weighted images within $72 \mathrm{~h}$ indicated

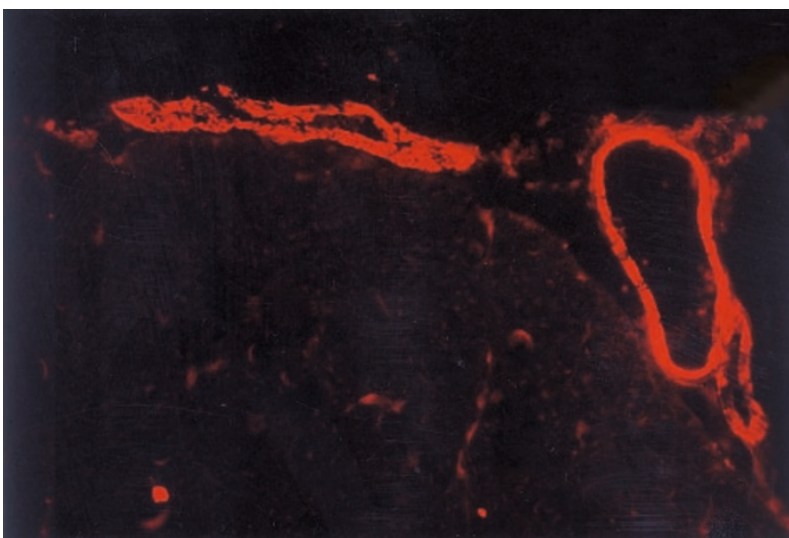

Figure 4 Examination for damage to the blood-spinal cord barrier in the dorsal column with Evans-blue showed that the dye had leaked out into the spinal cord parenchyma 2 days after the injury in the 20 -g compression group
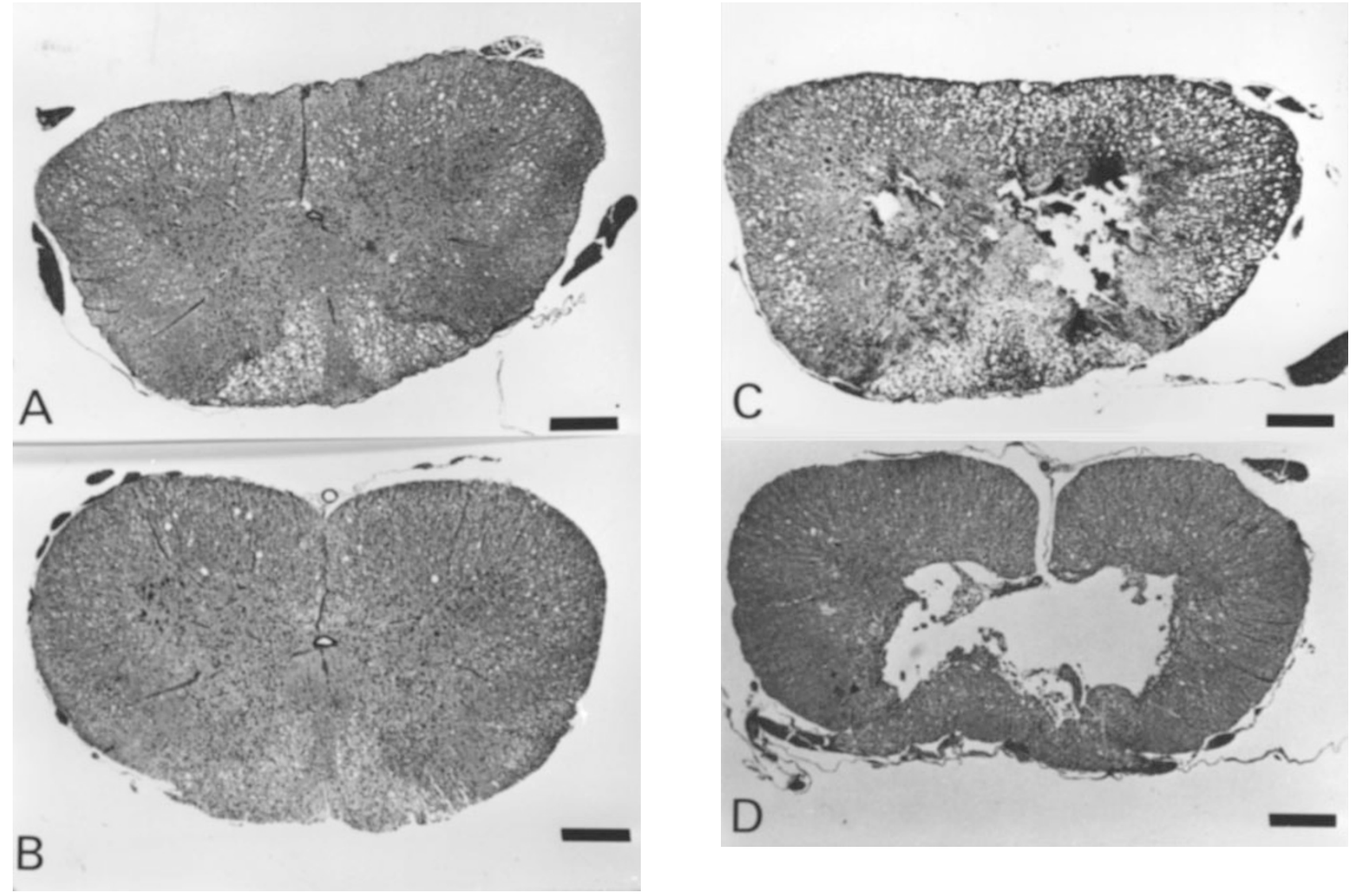

Figure 3 In the 20-g compression group, mild edema was observed 2 days after the injury (A) and reactive gliosis of the dorsal column was observed 28 days after the injury (B). In the 35 -g compression group, hemorrhagic lesions were presented in the gray matter 2 days after the injury $(\mathbf{C})$ and the cavity with a surrounding reactive gliosis was observed 28 days after the injury (D). (Staining with hematoxylin-eosin, scale bar $=300 \mu \mathrm{m}$ ) 


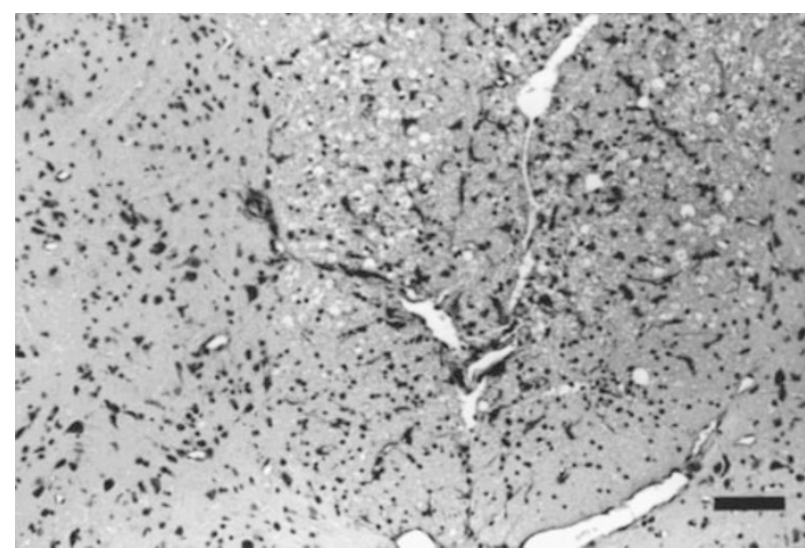

Figure 5 Staining by using GFAP antibody 28 days after the injury in the 20 -g compression group showed astrocyte proliferation primarily in the dorsal column. (Scale bar $=30 \mu \mathrm{m})$

hemorrhage within the spinal cord and that the prognosis was poor. Yamashita et $a l^{10}$ divided the MRI findings in the chronic stage of spinal cord injury into five patterns and reported poor improvement in symptoms in patients with low signals on T1-weighted images and high signals on T2-weighted images, and a poor prognosis in cases exhibiting signal changes within the cord on T2-weighted images in the acute stage. Some investigators ${ }^{19-13}$ interpreted the low signal areas among the early MRI signal changes after spinal cord injury on T2 images as representing deoxyhemoglobin in hemorrhage foci at sites of injury, and the high signal areas as representing edema.

Our results suggested that $\mathrm{T} 1$ iso signals and $\mathrm{T} 2$ diffuse high signal reflected edema due to damage to the blood-spinal cord barrier, 2 days after the injury in the 20-g compression group, which completely recovered from the paralysis after 28 days. In the $35-\mathrm{g}$ compression group, in which there was incomplete recovery from paralysis after 28 days, T1 iso signal/T2 low signal surrounded by a high signal 2 days after injury represented hemorrhage with surrounding edema. Moreover, T1 iso signal/T2 high signal after 28 days represented gliosis in the 20 -g compression group, and in the 35-g compression group $\mathrm{T} 1$ low signal/T2 high signal represented cavity formation.

Thus, T2 low signal of the spinal cord observed early after injury reflects hemorrhage and may serve as an indicator of a poor prognosis, T1 low signal and T2 high signal from the subacute to chronic period indicate persistence of paralysis and limited recovery of function.

\section{Conclusions}

A model of cervical cord injury was produced in 50 rats and MRI signal changes within the spinal cord were compared with the changes in motor dysfunction and histopathological changes. In the 20 -g compression group, MR images were T1 iso-signal/T2 high signal 2 days after the injury and T1 iso signal/T2 high signal after 28 days. The changes in MRI signal 2 and 28 days after the injury represented edema and gliosis, respectively. In the $35-\mathrm{g}$ compression group, MR images were $\mathrm{T} 1$ iso-signal and $\mathrm{T} 2$ low signal surrounded by high signal 2 days after the injury, and T1 low signal/T2 high signal after 28 days. The MRI signal changes 2 and 28 days after the injury reflected hemorrhage with edema and cavities, respectively.

\section{References}

1 Duncan EG et al. High-resolution magnetic-resonance imaging of experimental spinal cord injury in rat. J Neurosurg 1992; 31: $510-519$.

2 Hackney DB et al. Experimental spinal cord injury: imaging the acute lesion. AJNR 1994; 15: $960-961$.

3 Becerra JL et al. MR-Pathologic comparisons of Wallerian degeneration in spinal cord injury. $A J N R$ 1995; 16: $125-133$.

4 Holtz A, Nystrome B, Gerdin B. Spinal blood flow measured by ${ }^{14} \mathrm{C}$-iodoantipyrine autoradiography during and after graded spinal cord compression in rats. Surg Neurol 1989; 31: 350-360.

5 Nakamura $\mathrm{M}$ et al. Changes in choline acetyltransferase activity and distribution following incomplete cervical spinal cord injury in the rat. Neuroscience 1996; 75: 481-494.

6 Watanabe $\mathrm{M}$ et al. Changes of amino acid levels and aspartate distribtion in the cervical spinal cord after traumatic spinal cord injury. J Neurotrauma 1998; 15: 285-293.

7 Rivlin AS, Tator $\mathrm{CH}$. Effect of duration of acute spinal cord compression in a new acute spinal cord injury model in the rat. Surg Neurol 1978; 10: 39-43.

8 Shimada K, Tokioka T. Sequential MRI studies in patients with cervical cord injury but without bony injury. Paraplegia 1995; 33: $573-578$.

9 Kulkarni MV, McArdle CB. Acute spinal cord injury-MR imaging at 1.5T. Radiology 1987; 164: $837-843$.

10 Yamashita Y, Takahashi M. Chronic injuries of the spinal cord assessment with MR imaging. Radiology 1990; 175: 849-854.

11 Yamashita Y, Takahashi M. Acute spinal cord injury; Magnetic resonance imaging correlated with myelopathy. Br J Radiol 1991; 64: $201-209$.

12 Hackney DB, Asato R. Hemorrhage and edema in acute spinal cord compression demonstration by MR imaging. Radiology 1986; 161: $387-390$.

13 Weirich SD, Cotler HB. Histopathologic correlation of magnetic resonance imaging signal patterns in a spinal cord injury model. Spine 1990; 15: 630-638. 